\title{
DETERMINAÇÃO DO LIMIAR DE PERCEPÇÃO DE CORRENTE ELÉTRICA EM UMA AMOSTRA DE VOLUNTÁRIOS NORMAIS
}

\author{
Maria de Lourdes de Souza Galvão', Gilberto Mastrocola Manzano², \\ Nadia landoli de Oliveira Braga ${ }^{3}$, João Antonio Maciel Nóbrega ${ }^{4}$
}

\begin{abstract}
RESUMO - Determinaram valores de referência para limiares de percepção de corrente elétrica senoidal em indivíduos normais, utilizando-se o instrumento Neurometer ${ }^{\circledR}$; contrastaram-se os resultados com os fornecidos pelo fabricante; analisaram-se os limiares em diferentes segmentos corporais e avaliou-se o efeito da idade, sexo, grau de escolaridade e reutilização dos eletrodos. Estudou-se uma amostra de 101 voluntários normais (68 mulheres), com idades entre 17 e 87 anos. O procedimento foi completamente automatizado utilizando o método de límites e o de escolha forçada. Houve diferença significante entre os limiares obtidos no dedo indicador e no hálux nas três freqüências. Não houve diferenças significantes em relação ao sexo e grau de escolaridade; a idade apresentou discreta relação em algumas medidas. A reutilização dos eletrodos por três vezes nos dedos indicadores não comprometeu a confiabilidade, porém no hálux direito a reutilização por mais de duas vezes comprometeu a determinação do limiar para $2000 \mathrm{~Hz}$. Os valores obtidos diferiram das referências fornecidas pelo fabricante, nas freqüências $250 \mathrm{~Hz}$ e $5 \mathrm{~Hz}$.
\end{abstract}

PALAVRAS-CHAVE: limiar de percepção, corrente elétrica, valores de referência, sujeitos normais.

\begin{abstract}
Determination of electric current perception threshold in a sample of normal volunteers
ABSTRACT - Reference values using the instrument Neurometer ${ }^{\circledR}$ were determined and compared with those suggested by the manufacturer; the sensory thresholds for the different frequencies in different body segments were determined; the influence of age, level of education, gender and the reutilization of the electrodes in the different sites were also evaluated. Sensory threshold was determined in 101 normal volunteers ( 68 women), ages ranged from 17 to 87 years old. The procedure was fully automated with the sensory threshold being determined by the method of limits followed by the forced choice method. Analysis of the results revealed that: there was a significant difference between the values obtained for the index fingers and the halux; no difference was detected between both index fingers; gender and level of instruction had no effect on the results, age had a small influence after stimulation with $2000 \mathrm{~Hz}$ and $250 \mathrm{~Hz}$; three reutilizations of the electrodes did not affect the results on the index fingers, however at the halux, the third utilization deteriorated the results from the $2000 \mathrm{~Hz}$ stimulation. Except for the $2000 \mathrm{~Hz}$ stimulation, significant differences were suggested between the values reported here and those provided by the manufacturer.
\end{abstract}

KEY WORDS: sensory threshold, electric current, reference values, normal subjects.

A determinação do limiar sensitivo (LS), isto é, a menor quantidade de determinada energia capaz de evocar uma percepção, é uma medida importante para o estudo das vias sensoriais. A importância de sua utilização tem sido alvo de vários estudos que abordam diferentes metodologias de avaliação utilizando tanto estímulos físicos naturais quanto estímulos elétricos ${ }^{1,2}$. A utilização de estímulos elétricos para avaliação do LS teria a vantagem de ser mais facilmente controlável do ponto de vista prá- tico, porém, algumas dificuldades existem principalmente relacionadas ao comportamento não linear da impedância cutânea (que varia com a intensidade da estimulação elétrica) e a especificidade de estimulação de sistemas sensoriais distintos ${ }^{3}$. A introdução do equipamento Neurometer ${ }^{\circledast}$ (estimulador de corrente com programação automática para detecção de limiar de percepção de corrente elétrica) se propõe a resolver estes dois problemas, utilizando correntes elétricas senoidais de intensidade cons-

Setor de Neurofisiologia Clínica, Disciplina de Neurologia, Universidade Federal de São Paulo SP, Brasil (UNIFESP): ${ }^{1}$ Médica Aluna de Pós-Graduação; ${ }^{2}$ Professor Afiliado Doutor; ${ }^{3}$ Médica Assistente Doutora; ${ }^{4}$ Professor Adjunto Livre Docente. Estudo financiado pela FAPESP, como parte do projeto 01/05337-6.

Recebido 15 Julho 2004, recebido na forma final 22 Setembro 2004. Aceito 16 Novembro 2004.

Dr. Gilberto Mastrocola Manzano - Rua Dr. Thirso Martins 264 / 52 - 04120-050 São Paulo SP - Brasil. E-mail: gmmanzano@uol.com.br 
tante: a) a capacidade de variar a voltagem em função da variação da impedância cutânea manteria a intensidade de corrente aplicada constante (a variável que se correlacionaria à estimulação neural) e b) correntes senoidais permitiriam aumentar a seletividade da estimulação, isto é, diferentes freqüências estimulariam sistemas sensoriais relacionados com fibras de diferentes diâmetros de forma independente ${ }^{3,4}$. $O$ interesse em estudar novas alternativas para avaliar fibras sensitivas decorre da dificuldade em confirmar e mensurar disfunções sensitivas pelos métodos convencionais. Os estudos de condução nervosa constituem-se no método de escolha para avaliação das disfunções tanto sensitivas quanto motoras que acometem o sistema nervoso periférico ${ }^{3}$. Porém, sua principal limitação decorre da impossibilidade de avaliar as diferentes subpopulações de fibras sensitivas, pois o método só permite a avaliação das disfunções que acometem as fibras mielinizadas, especialmente aquelas de grande diâmetro ${ }^{5}$.

A existência de disfunções sensitivas que acometem fibras pouco mielinizadas e não mielinizadas, sem causar comprometimento simultâneo das fibras grossas mielinizadas, constitui-se na principal indicação clínica para a utilização de métodos de determinação do $\mathrm{LS}^{6,7}$.

Como etapa inicial da avaliação desta alternativa de determinação de $L S$, o objetivo deste estudo foi avaliar um equipamento estabelecendo os valores de referência para serem utilizados em nosso serviço e então contrastá-los com os resultados fornecidos pelo fabricante do equipamento, além de analisar a existência de diferenças entre os limiares em diferentes segmentos corporais, avaliar a influência de idade, sexo e grau de escolaridade, além de se analisar o efeito de reutilização do mesmo eletrodo em diferentes segmentos no mesmo indivíduo.

\section{MÉTODO}

Realizou-se um estudo prospectivo em 101 indivíduos normais que foram convidados a participar do estudo no setor de Neurofisiologia Clínica da Universidade Federal de São Paulo (UNIFESP - EPM) e no setor de Neurofisiologia Clínica do Hospital Santa Marcelina, no período de 05 de junho de 2002 a 22 de maio de 2003.

Critérios de inclusão - Indivíduos saudáveis, sem queixas sensitivas ou doenças que pudessem ocasionar comprometimento do sistema nervoso central ou periférico e sem história de traumatismos prévios relevantes.

Critérios de exclusão - Indivíduos com queixas sensitivas ou portadores de doenças que pudessem ocasionar comprometimento do sistema nervoso central ou periférico, tais como diabetes mellitus, distúrbios tireoidianos, doenças pulmonares crônicas, doença renal, uso de substâncias tóxicas, uso freqüente de álcool, deficiência visual e auditiva.

Os indivíduos foram submetidos ao teste sensitivo quantitativo utilizando o Neurometer ${ }^{\circledR}$ (modelo CPT/C, ano 2002) para determinação do limiar de percepção de corrente, nas freqüências de $5 \mathrm{~Hz}, 250 \mathrm{~Hz}$ e $2000 \mathrm{~Hz}$, a intensidades que poderiam variar de 0 a um máximo de 9,99 mA em 3 segmentos corpóreos pré-determinados, seguindo um protocolo totalmente automatizado. Os segmentos escolhidos foram o dedo indicador direito, o dedo indicador esquerdo e o hálux direito. A escolha desses segmentos foi motivada pelo interesse de estabelecer um intervalo de referência para os limiares, com o intuito de facilitar estudos posteriores em pacientes portadores de neuropatia periférica. Os resultados foram expressos em unidades CPT, onde um valor CPT de 001 equivale a $0,01 \mathrm{~mA}(10 \mu \mathrm{A})$.

Todos os indivíduos assinaram termo de consentimento informado, de acordo com projeto aprovado pelo Comitê de Ética e Pesquisa da UNIFESP-EPM. Procedeuse a interrogatório através de questionário para avaliação dos critérios de inclusão e exclusão e, a seguir, foram fornecidas as explicações a respeito do procedimento que seria realizado. Os indivíduos foram mantidos em uma sala reservada, com temperatura ambiente e acomodados confortavelmente.

A escolha do segmento a ser examinado inicialmente em cada um dos indivíduos selecionados ocorreu através de protocolo ordenado que obedeceu à ordem numérica de exame dos voluntários. O primeiro indivíduo foi examinado seguindo a ordem do primeiro protocolo que consistia em iniciar o teste de determinação do limiar pelo dedo indicador da mão direita, depois o dedo indicador da mão esquerda e finalmente o hálux direito. $O$ segundo indivíduo foi examinado seguindo a ordem do segundo protocolo que iniciava o teste pelo dedo indicador da mão esquerda, depois avaliava o hálux direito e então o indicador da mão direita. O terceiro indivíduo foi examinado seguindo a ordem do terceiro protocolo que iniciava o teste pelo hálux direito, sendo a seguir avaliado o dedo indicador da mão direita e por último o dedo indicador da mão esquerda. Os indivíduos seguintes foram avaliados pelos mesmos protocolos seguindo essa mesma ordem de testagem.

Análise estatística - 0 modelo estatístico envolveu uma análise de variância para medidas repetidas para avaliar diferenças entre os três segmentos corporais e entre as diferentes seqüências de testagem para avaliar o efeito da re-utilização de eletrodos. A partir desses resultados foi investigada a influência do sexo, da escolaridade e idade. As técnicas estatísticas utilizadas foram o teste $t$, a ANOVA ou análise de regressão de acordo com a natureza da variável envolvida. Como não foram 
detectadas diferenças significantes entre as variáveis de controle (sexo, escolaridade, idade), foi então investigada a distribuição das variáveis do segmento corporal e sua adesividade à distribuição normal (utilizando-se o teste de Shapiro-Wilk) e calculados os índices de assimetria e achatamento para, então, se construir os intervalos de referência. Após a determinação desses valores foi feita a avaliação destes resultados em relação àqueles propostos pelo fabricante do equipamento Neurometer ${ }^{\circledR}$, através da análise de gráficos de barras flutuantes. Nestes gráficos, os extremos de cada barra representam o valor da média adicionado (à direita) e subtraído (à esquerda) de duas vezes o erro padrão da respectiva média (para os dados fornecidos pelo fabricante estes valores foram calculados a partir dos valores fornecidos pelo mesmo); sua interpretação foi feita considerando-se que qualquer superposição dos intervalos sugere não haver diferença significante, o mesmo não ocorrendo para ausência de superposição. Na construção desses gráficos os valores para as freqüências de $5 \mathrm{~Hz}$ para os dedos indicadores foram calculados da mesma maneira, apesar da distribuição diferir significantemente da normal, somente com finalidade de comparação com os dados fornecidos pelo fabricante.

Diferenças significantes foram consideradas assumindo-se um risco $\alpha$ de 0,05 ou $5 \%$.

\section{RESULTADOS}

Durante a fase de coleta de dados foi observado que cinco indivíduos apresentaram valores limiares extremamente baixos (acentuadamente menores que o esperado pelos valores fornecidos pelo fabricante) e muito diferentes do segmento homólogo contralateral, no dedo indicador, especialmente na freqüência $5 \mathrm{~Hz}$ (ver abaixo). À inspeção local em três deles, foi observada a presença de algum pequeno trauma local mínimo, que não foi observada na inspeção inicial, embora em dois deles não tenha sido observada nenhuma lesão. Em virtude da assimetria acentuada dos valores, procedeu-se à realização de novo teste após uma ou duas semanas, para verificar a reprodutibilidade desses valores, quando então estas discrepâncias desapareceram e estes últimos valores foram então considerados nas análises; a média dos valores discrepantes iniciais foi 5,8 CPT (amplitude de variação de 2 a 13 CPT), após a segunda avaliação foi 65,4 CPT (amplitude de variação de 25 a 93 (PT), valores correspondentes no segmento homólogo foram 62,2 CPT (amplitude de variação 15 a 77 CPT).

Não foram detectadas diferenças significantes nos limiares para as diferentes freqüências, considerando-se sexo e graus de escolaridade; com relação à idade, foram detectadas correlações significantes
Tabela 1. Valor do limiar sensorial para as diferentes freqüencias nos diferentes segmentos expressos em unidades CPT (valores médios (Média) e desvios-padrões (DP)).

\begin{tabular}{lcc}
\hline & Média & DP \\
\hline $2000 \mathrm{~Hz}$ no indicador D & 232 & 53 \\
$2000 \mathrm{~Hz}$ no indicador E & 223 & 61 \\
$2000 \mathrm{~Hz}$ no hálux D & 324 & 62 \\
$250 \mathrm{~Hz}$ no indicador D & 87 & 36 \\
$250 \mathrm{~Hz}$ no indicador E & 82 & 33 \\
$250 \mathrm{~Hz}$ no hálux D & 141 & 38 \\
$5 \mathrm{~Hz}$ no indicador D & 59 & 32 \\
$5 \mathrm{~Hz}$ no indicador E & 63 & 32 \\
$5 \mathrm{~Hz}$ no hálux D & 83 & 36 \\
\hline
\end{tabular}

Tabela 2. Número de indivíduos (N), percentil 97,5 (Centil) e intervalos de confiança para os valores limiares (expressos em unidades (PT) obtidos nas diferentes freqüências.

\begin{tabular}{lcccc}
\hline & N & Centil & \multicolumn{2}{c}{$\begin{array}{c}\text { Intervalo de } \\
\text { confiança }\end{array}$} \\
\hline 2000Hz no indicador D & 101 & 330,0 & 306,73 & 352,87 \\
2000Hz no indicador E & 101 & 352,0 & 308,86 & 394,33 \\
2000Hz no hálux D & 67 & 461,0 & 393,51 & 528,09 \\
250Hz no indicador D & 101 & 169,4 & 132,02 & 206,88 \\
250Hz no indicador E & 101 & 152,4 & 129,34 & 175,56 \\
250Hz no hálux D & 101 & 216,3 & 195,76 & 236,94 \\
5Hz no indicador D & 101 & 135,0 & 90,30 & 179,30 \\
5Hz no indicador E & 101 & 143,0 & 88,25 & 198,35 \\
5Hz no hálux D & 101 & 151,0 & 133,65 & 167,95 \\
\hline
\end{tabular}

para a freqüência $2000 \mathrm{~Hz}$ nos segmentos indicador direito $(r=0,32 ; p=0,0010)$ e indicador esquerdo $(r=0,33 ; p=0,0007)$ e, para a freqüência $250 \mathrm{~Hz}$ no segmento indicador esquerdo $(r=0,32 ; p=0,0012)$. Porém, em nenhum dos casos a variação da idade explicou mais do que $11 \%$ da variabilidade dos dados. A comparação entre os segmentos revelou diferença significante nas diferentes freqüências estudadas ( $p<0,0001)$, não tendo sido detectada diferença significante entre os indicadores; porém, os valores para o hálux foram sempre significantemente maiores $(p<0,0001)$. A análise das seqüências de testagem só evidenciou diferença significante no estudo do limiar para $2000 \mathrm{~Hz}$ quando a terceira 


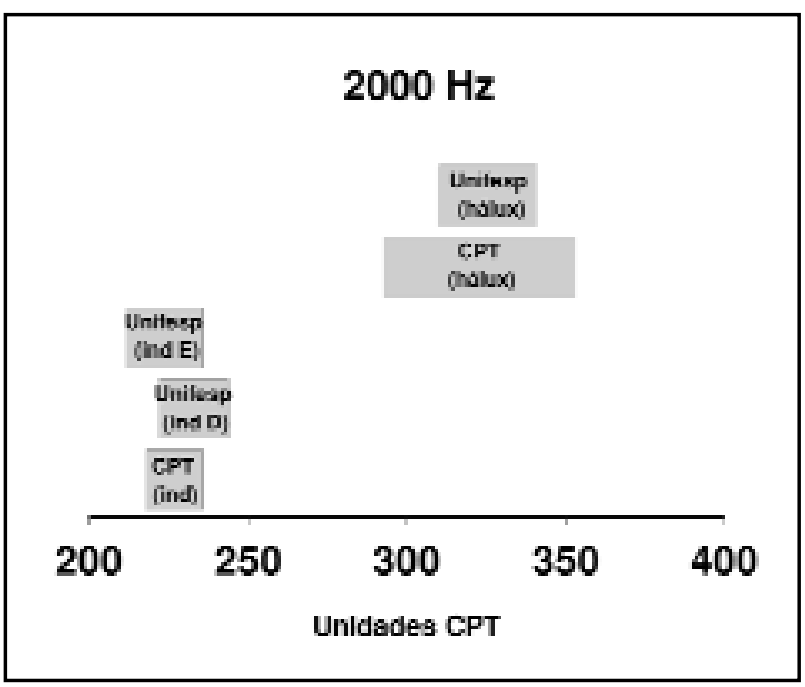

Fig 1. Intervalos de confiança para os valores médios dos limiares sensoriais para a freqüência de $2000 \mathrm{~Hz}$. CPT, valores oferecidos pelo fabricante; ind, dedo indicador; $D$, direito; $E$, esquerdo; UNIFESP, valores obtidos no presente estudo.

reutilização dos eletrodos ocorreu no hálux $(p=0,0019)$, motivo pelo qual os valores dos limiares obtidos no hálux para freqüência de $2000 \mathrm{~Hz}$, quando da terceira utilização dos eletrodos, foram excluídos das análises posteriores. Os valores dos limiares obtidos são mostrados na Tabela 1.

Após o agrupamento dos dados, procedeu-se à verificação da distribuição. Os dados somente diferiram significantemente da distribuição normal para a freqüência de $5 \mathrm{~Hz}$ nos segmentos dos dedos indicadores (indicador direito $p<0,0000$; indicador esquerdo $p<0,0311$ ).

Em seguida foram calculados os respectivos limites superiores dos intervalos de referência. Como limite superior do intervalo de referência foi então calculado o percentil 97,5 pelo método não paramétrico para a freqüência de $5 \mathrm{~Hz}$ para os segmentos dos dedos indicadores. Para as freqüências restantes e demais segmentos foi calculada a média mais 2 desvios-padrões. Os valores resultantes, bem como o intervalo de confiança (95\%), encontram-se na Tabela 2.

Os valores do presente estudo, assim como aqueles fornecidos pelo fabricante, estão mostrados nas Fig 1, 2 e 3 . Como pode ser visto, a sugestão de diferenças significantes ocorre para as freqüências $250 \mathrm{~Hz}$ e $5 \mathrm{~Hz}$.

\section{DISCUSSÃO}

Os resultados obtidos estão de acordo com os da literatura em relação à ausência de diferenças no valor limiar obtido, considerando as variáveis sexo e idade ${ }^{8}$. A diferença entre os valores obtidos

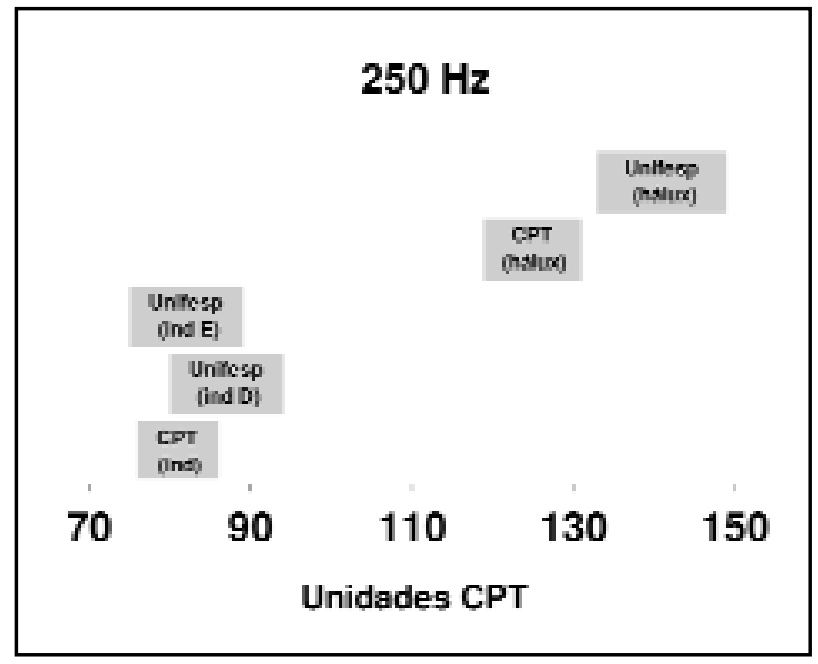

Fig 2. Intervalos de confiança para os valores médios dos limiares sensoriais para a freqüência de $250 \mathrm{~Hz}$. CPT; valores oferecidos pelo fabricante; ind, dedo indicador; $D$, direito; $E$, esquerdo; UNIFESP, valores obtidos no presente estudo.

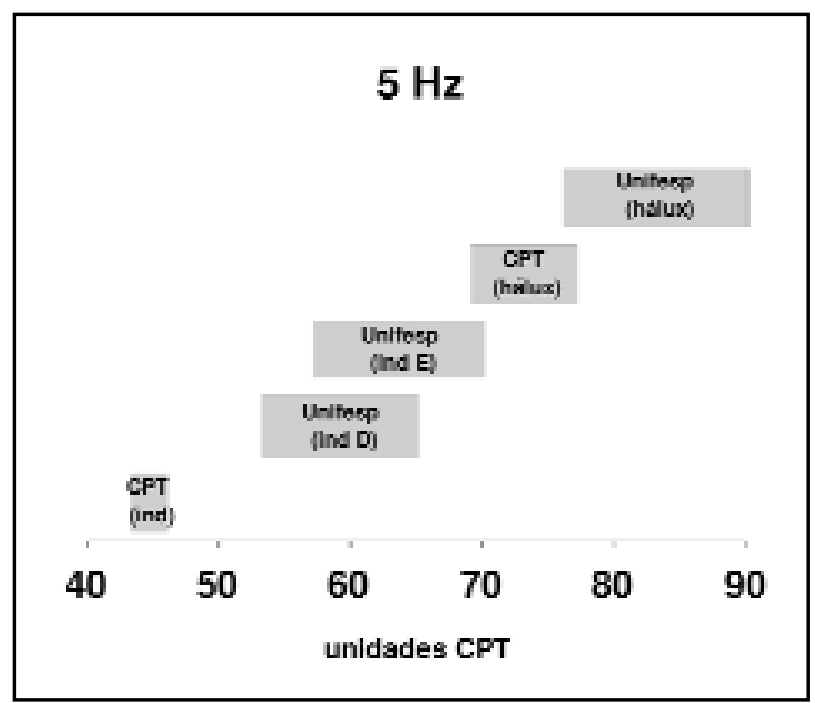

Fig 3. Intervalos de confiança para os valores médios dos limiares sensoriais para a freqüência de $5 \mathrm{~Hz}$. CPT, valores oferecidos pelo fabricante; ind, dedo indicador; $D$, direito; $E$, esquerdo; UNIFESP, valores obtidos no presente estudo.

nos dedos indicadores e no hálux também está de acordo com a literatura prévia ${ }^{8-10}$.

A reutilização do eletrodo por mais de duas vezes comprometeu a correta determinação do limiar no hálux para a freqüência de $2000 \mathrm{~Hz}$, mas o mesmo não ocorreu nos dedos indicadores. Tais achados sugerem que a reutilização de eletrodos em mais de dois locais no mesmo indivíduo altera significantemente os valores obtidos, não permitindo a comparação com os valores de referência. 
Valores muito baixos para limiares obtidos na freqüência $5 \mathrm{~Hz}$ têm sido descritos como indicadores de hiperpatia ${ }^{11}$, porém nossas observações sugerem que tal avaliação deva ser considerada somente após pelo menos uma repetição, com pelo menos uma semana de intervalo entre as determinações. A presença de pequenas soluções de continuidade nos locais de colocação dos eletrodos de estimulação, visíveis ou não, provavelmente criam vias de baixa resistência à passagem de corrente, prejudicando a avaliação deste parâmetro numa primeira mensuração; nossas observações sugerem que, em indivíduos saudáveis, o intervalo de uma semana é suficiente para o desaparecimento do efeito das mesmas.

Embora, do ponto de vista teórico, valores de referência devam ser sempre colhidos para cada laboratório, do ponto de vista prático algumas variáveis apresentam variabilidade tão baixa que, desde que se utilize rigorosamente a mesma metodologia, valores podem ser transpostos entre laboratórios. O contraste de nossos dados com os fornecidos pelo fabricante indica que a utilização do equipamento deva ser precedida por determinação de valores de referência para cada serviço, especialmente para baixas freqüências de estimulação.

A determinação dos valores limiares através da utilização do equipamento foi fácil, não apresentando nenhuma dificuldade especial. A capacidade de avaliação dos diferentes sistemas sensoriais através de correntes senoidais de diferentes freqüências ainda é assunto não definitivamente estabelecido e portanto a utilidade prática na avaliação de comprometimento dos diferentes sistemas sensoriais, especialmente aqueles relacionados a fibras finas, requer validação em pacientes, uma vez que sua utilidade nesta população é ainda controversa ${ }^{5,12}$. Neste sentido, é importante salientar que não foi detectada, neste estudo, influência importante da idade nos limiares sensoriais, o que está em desa- cordo com os achados utilizando-se formas mais naturais de estimulação ${ }^{3}$.

Em conclusão, a reutilização de eletrodos fornecidos pelo fabricante em mais de dois locais, no mesmo indivíduo, deve ser evitada, especialmente para $2000 \mathrm{~Hz}$ como freqüência de estimulação. A determinação de valores limiares para percepção de corrente elétrica, utilizando o equipamento estudado, não apresenta nenhuma dificuldade especial. Valores de referência devem ser obtidos em cada laboratório, não sendo recomendável a utilização dos valores propostos pelos fabricantes, especialmente para baixa freqüências. A interpretação de valores limiares abaixo do intervalo de referência para freqüência de $5 \mathrm{~Hz}$ deve ser considerada somente após pelo menos uma repetição, com pelo menos uma semana de intervalo entre as determinações.

\section{REFERÊNCIAS}

1. Dick PJ, O'Brien PC. Quantitative sensation testing in epidemiological and therapeutic studies of peripheral neuropathy. Muscle Nerve 1999; 22:659-662.

2. Wolfe GI. Quantitative sensory testing: basic principles and clinical applications. In Dumitru D (ed). Eletrodiagnostic medicine. 2nd edition. Philadelphia: Hanley \& Belfus, 2002: 429-437.

3. Yarnitsky D, Fowler CJ. Quantitative sensory testing. In: Osselton JW (ed). Clinical neurophysiology. Oxford: Butterworth-Heinemann, 1995: 253-270.

4. Neurometer CPT/C - Operating manual (version 17b), 2002:2.1-2.2.

5. Rendell MS, Katims JJ, Richter R, Rowland R. A comparison of nerve conduction velocities and current perception thresholds as correlates of clinical severity of diabetic sensory neuropathy. J Neurol Neurosurg Psychiatry 1989;52:502-511.

6. Lacomis D. Small-fiber neuropathy. Muscle Nerve 2002;26:173-188.

7. Tobin K, Giulliani MJ, Lacomis L. Comparision of different modalities for detection of small fiber neuropathy. Clin Neurophysiol 1999;110:1909-1912.

8. Evans ER, Rendell MS, Bartek JP, Bamisedun O, Connor S, Giitter M. Current perception thresholds in ageing. Age and Ageing 1992;21:273-279.

9. Katims JJ, Long DM, NG LKY. Transcutaneous nerve stimulation. Appl Neurophysiol 1986;49:86-91.

10. Rendell MS, Dovgan DJ, Bergman TF, O'Donnell GP, Drobny EP, Katims JJ. Mapping diabetic sensory neuropathy by current perception threshold testing. Diabetes Care 1989;12:636-640.

11. Neurometer CPT. Sensory nerve conduction threshold testing (sNCT): Neuval ${ }^{\circledast}$ Database II. Data Normative, 1999:1-7.

12. Tack CIJ, Netten PM, Scheepers MH, Meijer JWG, Smits P, Lutterman J. Comparison of clinical examination, current and vibratory perception threshold in diabetic polyneuropathy. Netherl J Med 1994;44:41-49. 